\title{
Partition function loop series for a general graphical model: free energy corrections and message-passing equations
}

\author{
Jing-Qing $\mathrm{Xiao}^{2,1}$ and Haijun Zhou ${ }^{1}$ \\ ${ }^{1}$ Key Laboratory of Frontiers in Theoretical Physics, Institute of Theoretical \\ Physics, Chinese Academy of Sciences, Beijing 100190, People's Republic of China \\ ${ }^{2}$ Institute of Applied Mathematics, Academy of Mathematics and Systems Science, \\ Chinese Academy of Sciences, Beijing 100190, People's Republic of China \\ E-mail: zhouhj@itp.ac.cn
}

\begin{abstract}
A loop series expansion for the partition function of a general statistical model on a graph is carried out. If the auxiliary probability distributions of the expansion are chosen to be a fixed point of the belief-propagation equation, the first term of the loop series gives the Bethe-Peierls free energy functional at the replicasymmetric level of the mean-field spin glass theory, and corrections are contributed only by subgraphs that are free of dangling edges. This result generalize the early work of Chertkov and Chernyak on binary statistical models. If the belief-propagation equation has multiple fixed points, a loop series expansion is performed for the grand partition function. The first term of this series gives the Bethe-Peierls free energy functional at the first-step replica-symmetry-breaking (RSB) level of the mean-field spin-glass theory, and corrections again come only from subgraphs that are free of dangling edges, provided that the auxiliary probability distributions of the expansion are chosen to be a fixed point of the survey-propagation equation. The same loop series expansion can be performed for higher-level partition functions, obtaining the higher-level RSB Bethe-Peierls free energy functionals (and the correction terms) and message-passing equations without using the Bethe-Peierls approximation.

PACS numbers: $05.50 .+\mathrm{q}, 02.10 . \mathrm{Ox}, 75.10 . \mathrm{Nr}$
\end{abstract}

Keywords: Bethe-Peierls free energy, belief-propagation, series expansion, partition function, replica-symmetry-breaking

Submitted to: J. Phys. A: Math. Gen. 


\section{Introduction}

Graph expansion methods for statistical models have been extensively discussed in the literature. They have been very helpful in studying the high-temperature behaviours and the phase transition properties of discrete models such as the Ising model of ferromagnetism. Some of the early efforts were carried out by Brout and others [1, 2, 3]. More recently, Georges and Yedidia [4] found that, high-temperature expansion of the Ising spin glass free energy can also be carried out under the constraints of fixed mean spin values. This later work was extended by Sessak and Monasson [5] to include pair correlations of spin variables as another set of expansion constraints. The constrained graph expansion method was applied to the inverse Ising problem [5], with the aim of inferring the microscopic interactions of a Ising system based on the observed mean spin values and spin-pair correlations.

For finite-connectivity binary statistical models, Chertkov and Chernyak [6, 7] showed that the partition function can be expressed as a sum of contributions from subgraphs. The first term of this expansion is identical to the partition function obtained using the Bethe-Peierls (BP) approximation. Loop corrections to the BP approximation was also calculated by Montanari, Rizzo, and collaborators [8, 9] and by Parisi and Slanina [10]. The derivation of the partition function expansion by Chertkov and Chernyak relied on a special property of Ising spin variables (see equation (17) of [7]). This special property is not valid for more general statistical models, whose microscopic variables are not necessarily binary or discrete. Whether the conclusion of [6, 7] is valid to general statistical models is still an open issue (for models whose discrete variables take $Q>2$ values, a complicated loop-tower expansion was presented in [11]).

In the present contribution we first extend the results of [6, 7, 11] by carrying out a very simple derivation of partition function loop series for a general statistical model defined on a graph. We do not make any assumptions on the nature of the microscopic state variable of each edge (or vertex) of the graph. This state variable can be discrete or real-valued, or be a vector, or even be a function itself. We show that the first term of this expansion is also identical to the Bethe-Peierls (BP) partition function, and corrections to the BP partition function come only from looped subgraphs without any dangling edges. The auxiliary probability distributions of this loop series expansion are chosen to be a fixed point of the belief-propagation equation. This particular choice makes all the subgraphs with at least one dangling edge to have zero contribution to the correction terms.

As the second main result, we present the loop series expressions for the grand partition function and higher-level partition functions. The belief-propagation equation of a statistical model may have multiple fixed points, each of which is referred to as a macroscopic state of the configuration space. If this happens, we define a grand partition function at the level of macroscopic states and perform a loop series expansion for this grand partition function. When the auxiliary probability distributions of this expansion 
are chosen to be a fixed point of the survey-propagation equation (first derived through the first-step replica-symmetry-breaking (1RSB) mean-field theory of spin glasses [12]), the first term of this loop expansion gives the BP free energy functional at the level of macroscopic states. Corrections again come only from subgraphs that are free of dangling edges. In case the survey-propagation equation has multiple fixed points, the same loop series expansion can be performed for higher-level partition functions. As a result, we obtain the higher-level BP free energy functionals and the correction contributions, and the associated message-passing equations.

This work is a mathematical approach to the theory of spin glasses from the framework of partition function loop expansion. It is clear that at each replicasymmetry-breaking (RSB) level of the mean-field theory [12] the corrections to the free energy due to looped nontrivial subgraphs are neglected. This neglected correction contribution is explicitly expressed as a logarithm over a finite series in this paper. At a given level of macroscopic states we anticipate that, the magnitude of the total loop correction contribution to the free energy will be sub-linear in $N$ ( $N$ being the total number of vertices) if there is only one fixed-point for the corresponding messagepassing equation, but it will be linear in $N$ if there exist multiple fixed-points. This statement needs to be checked by numerical calculations on single graphical systems.

Section 2 introduces the general statistical model. We work out the loop series of the partition function in Sec. 3 and derive the belief-propagation equation. In Sec. 4 we extend the discuss to the case that the belief-propagation equation has multiple fixed points, and perform a loop series expansion for the grand partition function. The 1RSB survey-propagation equation is derived here. We conclude this work in Sec. 5, and discuss some possible extensions. The Appendix A contains graph expansion results for a one-dimensional ring.

\section{General statistical models on graphs}

We consider a graph $G$ composed of $N$ vertices $(i=1,2, \ldots, N)$ and $M$ edges. An edge $(i, j)$ between two vertices $i$ and $j$ has a state variable $x_{i j}$. This variable may be a binary spin for some systems, $x_{i j}= \pm 1$. For other systems, $x_{i j}$ may be real-valued or be a vector, or be even more complicated. In this paper we make no assumptions on the nature of the microscopic state variable $x_{i j}$ of each edge $(i, j)$. Each vertex $i$ has an energy $E_{i}\left(x_{i \partial i}\right)$, where $x_{i \partial i} \equiv\left\{x_{i j_{1}}, x_{i j_{2}}, \ldots, x_{i j_{k}}\right\}$ with $j_{1}, j_{2}, \ldots, j_{k}$ being the $k$ other vertices with which $i$ forms an edge. The number $k$ of nearest neighbors of a vertex might be different for different vertices. The vertex energy is a function of the state variables of its connected edges. Notice that $x_{i j}$ and $x_{j i}$ both denote the state of edge $(i, j)$, therefore $x_{i j} \equiv x_{j i}$. The total vertex energy for an edge configuration $\left\{x_{i j}\right\}$ is

$$
E\left(\left\{x_{i j}\right\}\right)=\sum_{i=1}^{N} E_{i}\left(x_{i \partial i}\right) .
$$


The partition function of the system is defined as

$$
Z(\beta)=\prod_{(i, j)}\left[\int \mathrm{d} x_{i j} \rho_{0}\left(x_{i j}\right)\right] \prod_{i=1}^{N} e^{-\beta E_{i}\left(x_{i \partial i}\right)} .
$$

In the above equation, $\beta$ is the inverse temperature, $\rho_{0}\left(x_{i j}\right)$ is the probability of microscopic state $x_{i j}$ for an isolated edge $(i, j)$, and $\prod_{(i, j)}$ means the product over all the edges of graph $G$. For simplicity we assume that the a priori probabilities $\rho_{0}\left(x_{i j}\right)$ are identical for all edges. This assumption is of cause nonessential.

The partition function (2) also applies to graphical models whose microscopic states are defined on vertices rather than on edges [6, 7]. For example, consider a graph $G$ with the property that its vertices can be divided into two subsets, denoted by $\{i\}$ (the variable nodes) and $\{a\}$ (the check nodes), such that all the edges of $G$ are between a variable node $i$ and a check node $a$. For each variable node $i$ we assume that

$$
e^{-\beta E_{i}\left(x_{i \partial i}\right)}=\int \mathrm{d} x_{i} \rho_{0}\left(x_{i}\right) \prod_{a \in \partial i}\left[\frac{\delta\left(x_{i a}-x_{i}\right)}{\rho_{0}\left(x_{i a}\right)}\right],
$$

where $\partial i$ denotes the set of nearest neighboring check nodes of $i$. Equation (2) then simplifies to

$$
Z(\beta)=\prod_{i}\left[\int \mathrm{d} x_{i} \rho_{0}\left(x_{i}\right)\right] \prod_{a} e^{-\beta E_{a}\left(x_{\partial a}\right)},
$$

where $\partial a$ denotes the set of nearest neighboring variable nodes of $a$. Equation (4) is the partition function of a system defined on a factor graph, with each variable node $i$ having a microscopic state $x_{i}$ and each check node $a$ having an energy $E_{a}$. The check energy $E_{a}$ depends on the microscopic state $x_{\partial a}$ of the variable nodes in $\partial a$.

In some graphical models, the state $x_{i j}$ of each edge $(i, j)$ is a collection of two microscopic states $y_{i j}^{i}$ and $y_{i j}^{j}, x_{i j} \equiv\left\{y_{i j}^{i}, y_{i j}^{j}\right\}$. We assume that the a priori probability distribution of the edge state $x_{i j}$ equals to $\rho_{0}\left(y_{i j}^{i}\right) \rho_{0}\left(y_{i j}^{j}\right)$, and that the energy $E_{i}$ of a vertex $i$ can be expressed as

$$
e^{-\beta E_{i}\left(x_{i \partial i}\right)}=\int \mathrm{d} y_{i} \rho_{0}\left(y_{i}\right) e^{-\beta \tilde{E}_{i}\left(y_{i},\left\{y_{i j}^{j}\right\}\right)} \prod_{j \in \partial i}\left[\frac{\delta\left(y_{i}-y_{i j}^{i}\right)}{\rho_{0}\left(y_{i j}^{j}\right)}\right] .
$$

Under these assumptions, the partition function (2) becomes

$$
Z(\beta)=\prod_{i}\left[\int \mathrm{d} y_{i} \rho_{0}\left(y_{i}\right) e^{-\beta \tilde{E}_{i}\left(y_{i}, y_{\partial i}\right)}\right]
$$

which describes a graphical model whose vertex energy $\tilde{E}_{i}$ depends on the microscopic state $y_{i}$ of vertex $i$ and the microscopic states $y_{\partial i}$ of its nearest neighbors. An example of such statistical models is the palette-coloring problem [13, 14, 15].

\section{Graph expansion for the general statistical model}

To find a loop series expression for the partition function (2), we introduce for each edge $(i, j)$ two auxiliary probability distributions $q_{j \rightarrow i}\left(x_{i j}\right)$ and $q_{i \rightarrow j}\left(x_{j i}\right)$, and rewrite $Z(\beta)$ as

$$
Z(\beta)=\prod_{i=1}^{N} \prod_{j \in \partial i}\left[\int \mathrm{d} x_{i j} q_{j \rightarrow i}\left(x_{i j}\right)\right] e^{-\beta E_{i}\left(x_{i \partial i}\right)} \prod_{(k, l)} \frac{\delta\left(x_{k l}-x_{l k}\right) \rho_{0}\left(x_{k l}\right)}{q_{k \rightarrow l}\left(x_{l k}\right) q_{l \rightarrow k}\left(x_{k l}\right)}
$$


Loop series for general statistical models

$$
=\frac{1}{\prod_{(i, j)} C_{(i, j)}} \prod_{i=1}^{N} \prod_{j \in \partial i}\left[\int \mathrm{d} x_{i j} q_{j \rightarrow i}\left(x_{i j}\right)\right] e^{-\beta E_{i}\left(x_{i \partial i}\right)} \prod_{(k, l)}\left[1+\Delta_{(k, l)}\left(x_{k l}, x_{l k}\right)\right] .
$$

In (8), $C_{(i, j)}$ is an edge constant with the value

$$
C_{(i, j)}=\int \mathrm{d} x_{i j} \frac{q_{i \rightarrow j}\left(x_{i j}\right) q_{j \rightarrow i}\left(x_{i j}\right)}{\rho_{0}\left(x_{i j}\right)}
$$

and $\Delta_{(i, j)}\left(x_{i j}, x_{j i}\right)$ is expressed as

$$
\Delta_{(i, j)}\left(x_{i j}, x_{j i}\right) \equiv \frac{\delta\left(x_{i j}-x_{j i}\right) \rho_{0}\left(x_{i j}\right) C_{(i, j)}}{q_{i \rightarrow j}\left(x_{j i}\right) q_{j \rightarrow i}\left(x_{i j}\right)}-1 .
$$

From (8) we know that the partition function can be expressed as the sum of contributions from all the possible non-empty subgraphs of $G$ :

$$
Z(\beta)=Z_{B P}\left(1+\sum_{g \subseteq G} L_{g}\right) .
$$

In the above equation, $Z_{B P}$ is calculated by

$$
Z_{B P}=\frac{\prod_{i=1}^{N} \prod_{j \in \partial i}\left[\int \mathrm{d} x_{i j} q_{j \rightarrow i}\left(x_{i j}\right)\right] e^{-\beta E_{i}\left(x_{i \partial i}\right)}}{\prod_{(i, j)}\left[\int \mathrm{d} x_{i j} \frac{q_{i \rightarrow j}\left(x_{i j}\right) q_{j \rightarrow i}\left(x_{i j}\right)}{\rho_{0}\left(x_{i j}\right)}\right]} .
$$

A non-empty subgraph $g$ of graph $G$ contains a partial set of the edges of $G$ and all the vertices that are attached to these edges. The correction $L_{g}$ is expressed as

$$
L_{g}=\prod_{i \in g} \frac{\prod_{j \in \partial i}\left[\int \mathrm{d} x_{i j} q_{j \rightarrow i}\left(x_{i j}\right)\right] e^{-\beta E_{i}\left(x_{i \partial i}\right)}}{\prod_{j \in \partial i}\left[\int \mathrm{d} y_{i j} q_{j \rightarrow i}\left(y_{i j}\right)\right] e^{-\beta E_{i}\left(y_{i \partial i}\right)}} \prod_{(k, l) \in g} \Delta_{(k, l)}\left(x_{k l}, x_{l k}\right) .
$$

Consider a subgraph $g$ which has a vertex $i$ that is linked to the other parts of $g$ only through a single edge $(i, j)$. The neighborhood of such a leaf vertex $i$ is shown schematically in figure 1. We find that after integrating over the variable $x_{i j}$, the correction $L_{g}$ is expressed as

$$
\begin{aligned}
L_{g}=\prod_{k \in g \backslash i} \frac{\prod_{l \in \partial k}\left[\int \mathrm{d} x_{k l} q_{l \rightarrow k}\left(x_{k l}\right)\right] e^{-\beta E_{k}\left(x_{k \partial k}\right)}}{\prod_{l \in \partial k}\left[\int \mathrm{d} y_{k l} q_{l \rightarrow k}\left(y_{k l}\right)\right] e^{-\beta E_{k}\left(y_{k \partial k}\right)}} \prod_{(m, n) \in g \backslash(i, j)} \Delta_{(m, n)}\left(x_{m n}, x_{n m}\right) \\
\quad \times\left\{\frac{\hat{q}_{i \rightarrow j}\left(x_{j i}\right) \int \mathrm{d} y_{i j} q_{j \rightarrow i}\left(y_{i j}\right) q_{i \rightarrow j}\left(y_{i j}\right) / \rho_{0}\left(y_{i j}\right)}{q_{i \rightarrow j}\left(x_{j i}\right) \int \mathrm{d} y_{i j} q_{j \rightarrow i}\left(y_{i j}\right) \hat{q}_{i \rightarrow j}\left(y_{i j}\right) / \rho_{0}\left(y_{i j}\right)}-1\right\},
\end{aligned}
$$

where $\hat{q}_{i \rightarrow j}\left(x_{i j}\right)$ is determined by the set of probability functions $q_{\partial i \backslash j} \equiv\left\{q_{k \rightarrow i}, k \in \partial i \backslash j\right\}$ through

$$
\hat{q}_{i \rightarrow j}\left(x_{i j}\right)=B_{i \rightarrow j}\left(q_{\partial i \backslash j}\right) \equiv \frac{\rho_{0}\left(x_{i j}\right) \prod_{k \in \partial i \backslash j}\left[\int \mathrm{d} x_{i k} q_{k \rightarrow i}\left(x_{i k}\right)\right] e^{-\beta E_{i}\left(x_{i \partial i}\right)}}{\int \mathrm{d} y_{i j} \rho_{0}\left(y_{i j}\right) \prod_{k \in \partial i \backslash j}\left[\int \mathrm{d} y_{i k} q_{k \rightarrow i}\left(y_{i k}\right)\right] e^{-\beta E_{i}\left(y_{i \partial i}\right)}} .
$$

The function $B_{i \rightarrow j}\left(q_{\partial i \backslash j}\right)$ as defined by (15) is called the belief-propagation equation. It takes as input a set of probability distributions $q_{k \rightarrow i}\left(x_{i k}\right)(k \in \partial i)$ and outputs a new probability distribution $\hat{q}_{i \rightarrow j}\left(x_{i j}\right)$.

Since we are free to choose the auxiliary probabilities functions $\left\{q_{i \rightarrow j}\left(x_{j i}\right)\right\}$, we can choose this set of auxiliary functions to be a fixed point of the belief-propagation 


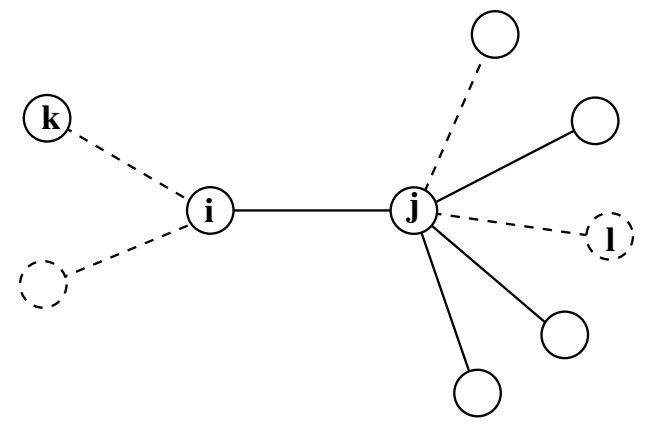

Figure 1. The neighborhood of a vertex $i$. A solid line between any two vertices $i$ and $j$ means that the edge $(i, j)$ is presented both in graph $G$ and in subgraph $g$. A dashed line between two vertices $i$ and $k$ means that the edge $(i, k)$ is presented in $G$ but not in $g$. A solid circle denotes a vertex that belongs to subgraph $g$, and a dashed circle denotes a vertex that is not belonging to $g$. A solid circle is attached by at least one solid edge. In this figure, vertex $i$ is connected by only one solid edge, it is a leaf vertex of subgraph $g$, and the edge $(i, j)$ is a dangling edge.

equation 15). In other words, we require that the auxiliary probability functions to satisfy

$$
q_{i \rightarrow j}\left(x_{i j}\right)=B_{i \rightarrow j}\left(\left\{q_{k \rightarrow i}\left(x_{i k}\right), k \in \partial i \backslash j\right\}\right) .
$$

Then for each edge $(i, j)$ we have $\hat{q}_{i \rightarrow j}\left(x_{i j}\right)=q_{i \rightarrow j}\left(x_{i j}\right)$, and the expression inside the curly brackets of (14) is identically zero. Under this special choice, only those subgraphs of graph $G$ with each vertex $i$ having at least two attached edges have nonzero contributions to the correction of the partition function. The total free energy $F(\beta)$ is then expressed as

$$
F(\beta) \equiv-\frac{1}{\beta} \ln Z(\beta)=F_{B P}(\beta)-\frac{1}{\beta} \ln \left[1+\sum_{g^{\prime} \subseteq G} L_{g^{\prime}}\right],
$$

where $g^{\prime}$ denotes a looped subgraph that contains no dangling edges. The free energy $F_{B P}(\beta)$ corresponds to the partition function $Z_{B P}$ and is expressed as

$$
F_{B P}(\beta)=\sum_{i} f_{i}-\sum_{(i, j)} f_{(i, j)},
$$

with

$$
\begin{aligned}
f_{i} & =-\frac{1}{\beta} \ln \left[\prod_{k \in \partial i}\left[\int \mathrm{d} x_{i k} q_{k \rightarrow i}\left(x_{i k}\right)\right] e^{-\beta E_{i}\left(x_{i \partial i}\right)}\right], \\
f_{(i, j)} & =-\frac{1}{\beta} \ln \left[\int \mathrm{d} x_{i j} \frac{q_{i \rightarrow j}\left(x_{i j}\right) q_{j \rightarrow i}\left(x_{i j}\right)}{\rho_{0}\left(x_{i j}\right)}\right] .
\end{aligned}
$$

We emphasize that $F_{B P}(\beta)$ is identical in form to the mean-field free energy as obtained by the replica-symmetric (RS) spin-glass theory [12. The expression (18) was first derived in the mean-field theory by using the BP approximation. The free energy $F_{B P}$ can also be viewed as a functional of the $2 M$ probability distributions $\left\{p_{i \rightarrow j}\left(x_{i j}\right)\right\}$ on the $M$ edges $(i, j)$ of graph $G$. In this paper we refer $F_{B P}$ as the BP free energy 
functional. It is easy to check that the variation of $F_{B P}$ with respect to any a probability distribution $p_{i \rightarrow j}\left(x_{i j}\right)$ is zero at a fixed point of (16). Equation (18) is expressed as the difference between the total vertex contribution $\left(\sum_{i} f_{i}\right)$ and the total edge contribution $\left(\sum_{(i, j)} f_{(i, j)}\right)$. An intuitive understanding of this is as follows: Each edge participates in two vertex interactions and its effect is counted twice when calculating the total vertex contribution; this over-counting should be subtracted from the total vertex contribution.

From the viewpoint of partition function loop expansion, the belief-propagation fixed-point condition (16) is a requirement for ensuring all the corrections $L_{g}$ from subgraphs $g$ with dangling edges are identically zero. For a loopy subgraph $g$ without dangling edges, its correction contribution $L_{g}$ is obtained through (13). The correction to the total free energy is expressed as the logarithm of the sum of all these loop correction contributions [see (17)]. In Appendix A we report the free energy correction contribution of a one-dimensional ring of $N$ edges. The correction is found to be positive when this ring is energetically frustrated. For more complicated model systems, the sign of the free energy correction contribution is still an open issue.

For a discrete model whose edge states can take $Q>2$ different values, Chernyak and Chertkov [11] derived a loop-tower expansion for the partition function by exploiting the gauge symmetry of the microscopic states. The derived belief-propagation equation by their approach does not fix the gauge freedom completely, and therefore high-order gauge fixing was introduced, making the loop-tower expansion scheme very complicated. Gauge fixing is not needed in the present loop series expansion scheme. In light of the present work, it might be possible to simplify the scheme of [11] and get an alternative derivation of the free energy expression (17). We are currently working on this mathematical issue.

\section{Graph expansion for the grand partition function}

For the general statistical model defined by the partition function (2), the beliefpropagation equation (16) may have multiple fixed points. If this happens, then the BP free energy $F_{B P}$ as a functional of $\left\{p_{i \rightarrow j}\left(x_{i j}\right)\right\}$ has more than one minimal value. In the following, we will refer to a fixed-point solution $\left\{p_{i \rightarrow j}\left(x_{i j}\right)\right\}$ of $(16)$ with a minimal value of $F_{B P}$ as a macroscopic state of the configuration space. Each macroscopic state has a corresponding BP free energy value $F_{B P}$. To account for the existence of multiple macroscopic states, in analogy with (2), we define a grand partition function $\Xi$ as

$\Xi=\prod_{(i, j)}\left[\iint \mathrm{D} q_{i \rightarrow j} \mathrm{D} q_{j \rightarrow i} \delta\left(q_{i \rightarrow j}-B_{i \rightarrow j}\left(q_{\partial i \backslash j}\right)\right) \delta\left(q_{j \rightarrow i}-B_{j \rightarrow i}\left(q_{\partial j \backslash i}\right)\right)\right] \exp \left(-y F_{B P}\right)$.

In the above equation, $\int \mathrm{D} q_{i \rightarrow j}$ means summing over all different possibilities of the distribution $q_{i \rightarrow j}$, and the Dirac delta functions $\delta\left(q_{i \rightarrow j}-B_{i \rightarrow j}\left(q_{\partial i \backslash j}\right)\right)$ ensure that only fixed-point solutions of the belief-propagation equation (16) contribute to $\Xi$. The parameter $y$ is an introduced inverse temperature at the level of macroscopic states. 
In analogy with (7) we can rewrite (21) as

$$
\Xi=\frac{1}{\prod_{(i, j)} C_{(i, j)}^{(1)}} \prod_{i=1}^{N}\left[\prod_{j \in \partial i} \int \mathrm{D} q_{j \rightarrow i} P_{j \rightarrow i}\left(q_{j \rightarrow i}\right) e^{-y f_{i}}\right] \prod_{(k, l)}\left[1+\Delta_{(k, l)}^{(1)}\right] .
$$

In the above equation, $P_{i \rightarrow j}\left(q_{i \rightarrow j}\right)$ is an introduced auxiliary probability distribution function for the probability distribution $q_{i \rightarrow j}\left(x_{j i}\right) ; f_{i}$ is the free energy contribution of vertex $i$ as expressed by $(19) ; C_{(i, j)}^{(1)}$ is an edge constant,

$$
C_{(i, j)}^{(1)}=\iint \mathrm{D} q_{i \rightarrow j} \mathrm{D} q_{j \rightarrow i} P_{i \rightarrow j}\left(q_{i \rightarrow j}\right) P_{j \rightarrow i}\left(q_{j \rightarrow i}\right) e^{-y f_{(i, j)}},
$$

with $f_{(i, j)}$ being the free energy contribution of an edge $(i, j)$ as given by 20$)$; and $\Delta_{(i, j)}^{(1)}$ is expressed as

$$
\Delta_{(i, j)}^{(1)} \equiv \frac{\delta\left(q_{i \rightarrow j}-B\left(q_{\partial i \backslash j}\right)\right) \delta\left(q_{j \rightarrow i}-B\left(q_{\partial j \backslash i}\right)\right) C_{(i, j)}^{(1)}}{P_{i \rightarrow j}\left(q_{i \rightarrow j}\right) P_{j \rightarrow i}\left(q_{j \rightarrow i}\right) e^{-y f_{(i, j)}}}-1 .
$$

The grand partition function can therefore be expanded as

$$
\Xi=\Xi_{S P}\left(1+\sum_{g \subseteq G} L_{g}^{(1)}\right),
$$

where $\Xi_{S P}$ is expressed as

$$
\Xi_{S P}=\frac{\prod_{i=1}^{N}\left[\prod_{j \in \partial i} \int \mathrm{D} q_{j \rightarrow i} P_{j \rightarrow i}\left(q_{j \rightarrow i}\right) e^{-y f_{i}}\right]}{\prod_{(i, j)}\left[\iint \mathrm{D} q_{i \rightarrow j} \mathrm{D} q_{j \rightarrow i} P_{i \rightarrow j}\left(q_{i \rightarrow j}\right) P_{j \rightarrow i}\left(q_{j \rightarrow i}\right) e^{-y f_{(i, j)}}\right]},
$$

and the correction term $L_{g}^{(1)}$ of a subgraph $g$ is expressed as

$$
L_{g}^{(1)}=\prod_{i \in g} \frac{\prod_{j \in \partial i}\left[\int \mathrm{D} q_{j \rightarrow i} P_{j \rightarrow i}\left(q_{j \rightarrow i}\right)\right] e^{-\beta f_{i}\left(\left\{q_{j \rightarrow i}\right\}\right)}}{\prod_{j \in \partial i}\left[\int \mathrm{D} p_{j \rightarrow i} P_{j \rightarrow i}\left(p_{j \rightarrow i}\right)\right] e^{-\beta f_{i}\left(\left\{p_{j \rightarrow i}\right\}\right)}} \prod_{(k, l) \in g} \Delta_{(k, l)}^{(1)} .
$$

Consider a subgraph $g$ which has a leaf vertex $i$ and a dangling edge $(i, j)$. After integrating over the probabilities around vertex $i$, the correction contribution of this subgraph can be expressed as

$$
\begin{aligned}
L_{g}^{(1)}= & \prod_{k \in g \backslash i} \frac{\prod_{l \in \partial k}\left[\int \mathrm{D} q_{l \rightarrow k} P_{l \rightarrow k}\left(q_{l \rightarrow k}\right)\right] e^{-\beta f_{k}\left(\left\{q_{l \rightarrow k}\right\}\right)}}{\prod_{l \in \partial k}\left[\int \mathrm{D} p_{l \rightarrow k} P_{l \rightarrow k}\left(p_{l \rightarrow k}\right)\right] e^{-\beta f_{k}\left(\left\{p_{l \rightarrow k}\right)\right\}}} \prod_{(m, n) \in g \backslash(i, j)} \Delta_{(m, n)}^{(1)} \\
& \times\left\{\frac{\hat{P}_{i \rightarrow j}\left(q_{i \rightarrow j}\right) \iint \mathrm{D} q_{i \rightarrow j} \mathrm{D} q_{j \rightarrow i} P_{i \rightarrow j}\left(q_{i \rightarrow j}\right) P_{j \rightarrow i}\left(q_{j \rightarrow i}\right) e^{-y f_{(i, j)}}}{P_{i \rightarrow j}\left(q_{i \rightarrow j}\right) \iint \mathrm{D} q_{i \rightarrow j} \mathrm{D} q_{j \rightarrow i} \hat{P}_{i \rightarrow j}\left(q_{i \rightarrow j}\right) P_{j \rightarrow i}\left(q_{j \rightarrow i}\right) e^{-y f_{(i, j)}}}-1\right\},
\end{aligned}
$$

where the probability distribution $\hat{P}_{i \rightarrow j}\left(q_{i \rightarrow j}\right)$ is calculated by

$$
\hat{P}_{i \rightarrow j}\left(q_{i \rightarrow j}\right)=\frac{\prod_{k \in \partial i \backslash j}\left[\int \mathrm{D} q_{k \rightarrow i} P_{k \rightarrow i}\left(q_{k \rightarrow i}\right)\right] e^{-y f_{i \rightarrow j}} \delta\left(q_{i \rightarrow j}-B_{i \rightarrow j}\left(q_{\partial i \backslash j}\right)\right)}{\prod_{k \in \partial i \backslash j}\left[\int \mathrm{D} q_{k \rightarrow i} P_{k \rightarrow i}\left(q_{k \rightarrow i}\right)\right] e^{-y f_{i \rightarrow j}}},
$$

with

$$
f_{i \rightarrow j}=-\frac{1}{\beta} \ln \left[\int \mathrm{d} x_{i j} \rho_{0}\left(x_{i j}\right) \prod_{k \in \partial i \backslash j} \int \mathrm{d} x_{i k} q_{k \rightarrow i}\left(x_{i k}\right) e^{-\beta E_{i}\left(x_{i \partial i}\right)}\right] .
$$

In accordance with the spin-glass literature, we refer (29) as the the survey-propagation equation. 
The expression inside the curly brackets of 28 is identically zero if $\hat{P}_{i \rightarrow j}\left(q_{i \rightarrow j}\right)=$ $P_{i \rightarrow j}\left(q_{i \rightarrow j}\right)$. Since the auxiliary probability distributions $\left\{P_{i \rightarrow j}\left(q_{i \rightarrow j}\right)\right\}$ are free to choose, we can choose them appropriately to ensure that the correction contribution $L_{g}^{(1)}=0$ for any a subgraph $g$ with at least one dangling edge. In other words, $\left\{P_{i \rightarrow j}\left(q_{i \rightarrow j}\right)\right\}$ should be a fixed-point solution of the survey-propagation equation:

$$
P_{i \rightarrow j}\left(q_{i \rightarrow j}\right)=\frac{\prod_{k \in \partial i \backslash j}\left[\int \mathrm{D} q_{k \rightarrow i} P_{k \rightarrow i}\left(q_{k \rightarrow i}\right)\right] e^{-y f_{i \rightarrow j}} \delta\left(q_{i \rightarrow j}-B_{i \rightarrow j}\left(q_{\partial i \backslash j}\right)\right)}{\prod_{k \in \partial i \backslash j}\left[\int \mathrm{D} q_{k \rightarrow i} P_{k \rightarrow i}\left(q_{k \rightarrow i}\right)\right] e^{-y f_{i \rightarrow j}}} .
$$

This equation was first derived in [12] under physical considerations (the BP approximation was again used).

At a fixed point of (31), the expression of the total grand free energy is

$$
G(y ; \beta) \equiv-\frac{1}{y} \ln \Xi=G_{S P}(y ; \beta)-\frac{1}{y} \ln \left[1+\sum_{g^{\prime} \subseteq G} L_{g^{\prime}}^{(1)}\right],
$$

where $g^{\prime}$ again denotes a looped subgraph that contains no dangling edges. From the framework of partition function loop expansion, (31) is a requirement to ensure that subgraphs with dangling edges do not have correction contributions to the grand free energy.

In (32), the grand free energy $G_{S P}(y ; \beta)$ is expressed as

$$
G_{S P}(y ; \beta) \equiv-\frac{1}{y} \ln \Xi_{S P}=\sum_{i} g_{i}-\sum_{(i, j)} g_{(i, j)},
$$

with

$$
\begin{aligned}
g_{i} & =-\frac{1}{y} \ln \left[\prod_{j \in \partial i} \int \mathrm{D} q_{j \rightarrow i} P_{j \rightarrow i}\left(q_{j \rightarrow i}\right) e^{-y f_{i}}\right], \\
g_{(i, j)} & =-\frac{1}{y} \ln \left[\iint \mathrm{D} q_{i \rightarrow j} \mathrm{D} q_{j \rightarrow i} P_{i \rightarrow j}\left(q_{i \rightarrow j}\right) P_{j \rightarrow i}\left(q_{j \rightarrow i}\right) e^{-y f_{(i, j)}}\right]
\end{aligned}
$$

being, respectively, the contribution to the grand free energy from a vertex $i$ and an edge $(i, j) . G_{S P}(y ; \beta)$ is identical in form to the 1RSB free energy of the mean-field spin-glass theory [12, which was derived previously by applying the BP approximation. $G_{S P}$ can also be regarded as a functional of the $2 M$ probabilities $\left\{P_{i \rightarrow j}\left(q_{i \rightarrow j}\right)\right\}$, and its variation with respect to any a $P_{i \rightarrow j}\left(q_{i \rightarrow j}\right)$ is zero at a fixed-point of the surveypropagation equation. We refer $G_{S P}$ as the survey-propagation free energy functional (it is the BP free energy functional at the 1RSB mean-field level).

We end this section with a discussion on the reweighting parameter $y$ of (21). Denoting a fixed-point solution of the belief-propagation equation (a macroscopic state) as $\alpha$ and its associated BP free energy as $F_{B P}^{(\alpha)}$, the grand partition function $\Xi$ can be re-written as

$$
\Xi=\sum_{\alpha} \exp \left(-y F_{B P}^{(\alpha)}\right)=\int \mathrm{d} f \exp [N(\Sigma(f)-y f)]
$$

where $\exp (N \Sigma(f))$ is the total number of macroscopic states with a given BP free energy $F_{B P}=N f$ (the quantity $f$ is called the free energy density). The function $\Sigma(f)$ is called the complexity in the spin-glass literature (it is the entropy density at the level 
of macroscopic states). For $N \gg 1$, the integration in (36) are dominated by the value of $f=\bar{f}$ which satisfies $\left.\frac{\mathrm{d} \Sigma(f)}{\mathrm{d} f}\right|_{f=\bar{f}}=y$. The value $\bar{f}$ is the mean BP free energy density at a given value of $y$. If we neglect the loop correction to the grand free energy in (32), then

$$
N \bar{f} \approx \frac{\partial\left[y G_{S P}(y ; \beta)\right]}{\partial y}=\sum_{i} \bar{f}_{i}-\sum_{(i, j)} \bar{f}_{(i, j)},
$$

where $\bar{f}_{i}$ and $\bar{f}_{(i, j)}$ are, respectively, the mean free energy contribution of a vertex $i$ and an edge $(i, j)$, with the expression

$$
\begin{aligned}
\bar{f}_{i} & =\frac{\prod_{j \in \partial i} \int \mathrm{D} q_{j \rightarrow i} P_{j \rightarrow i}\left(q_{j \rightarrow i}\right) f_{i} e^{-y f_{i}}}{\prod_{j \in \partial i} \int \mathrm{D} q_{j \rightarrow i} P_{j \rightarrow i}\left(q_{j \rightarrow i}\right) e^{-y f_{i}}}, \\
\bar{f}_{(i, j)} & =\frac{\iint \mathrm{D} q_{i \rightarrow j} \mathrm{D} q_{j \rightarrow i} P_{i \rightarrow j}\left(q_{i \rightarrow j}\right) P_{j \rightarrow i}\left(q_{j \rightarrow i}\right) f_{(i, j)} e^{-y f_{(i, j)}}}{\iint \mathrm{D} q_{i \rightarrow j} \mathrm{D} q_{j \rightarrow i} P_{i \rightarrow j}\left(q_{i \rightarrow j}\right) P_{j \rightarrow i}\left(q_{j \rightarrow i}\right) e^{-y f_{(i, j)}}} .
\end{aligned}
$$

The value of the complexity $\Sigma$ is expressed as

$$
\Sigma=y\left[\bar{f}-\frac{1}{N} G_{S P}(y ; \beta)\right]
$$

The smallest mean free energy density $\bar{f}$ corresponds to the value of $y$ which makes the complexity be zero, $\Sigma=0$. Another special value of $y$ is $y=\beta$. If the complexity calculated at $y=\beta$ is positive, the corresponding mean free energy density value $\bar{f}$ is the typical value of BP free energy densities of the macroscopic states sampled at inverse temperature $\beta[12]$.

\section{Conclusion and discussion}

The main results of this paper are the free energy expression (17) and the grand free energy expression (32), and the corresponding belief-propagation equation (16) and survey-propagation equation (31). From the viewpoint of partition function loop expansion, the belief-propagation and survey-propagation equation are, respectively, conditions needed to ensure that subgraphs with dangling edges have zero correction contributions to the free energy and the grand free energy.

This work helps to place the mean-field RSB theory of spin glasses on a firmer mathematical ground. There are many unsolved problems ahead. For example, the relationship free energy functionals $G_{B P}$ and $G_{S P}$ and the free energy landscape of the system; the link between the defined grand partition function $\Xi$ and the original partition function $Z$; the issue of sampling microscopic configurations $\left\{x_{i j}\right\}$ giving a fixed-point solution $\left\{q_{j \rightarrow i}\left(x_{i j}\right)\right\}$ of the belief-propagation equation; and son on.

The discussion in Sec. 4 can be readily extended to the case that the surveypropagation equation (31) has multiple fixed-point solutions. As a result, the mean-field second-step RSB free energy and its loop correction expression will be derived, as well as the corresponding message-passing equation. This expansion hierarchy can be continued to produce the mean-field results and the corresponding loop correction expressions and message-passing equations at even higher-levels of replica-symmetry-breaking. 
For statistical models defined on a factor graph with partition functions expressed in the form of (4), the method of this paper can also be directly applied without the need of first turning the partition function into the form of (2).

The present paper also points to some other important open issues. One question is: How to express the mean value of a local observable in terms of a finite loop series? Examples of local observables are the state variable $x_{i j}$ on an edge $(i, j)$ of the system, the correlation between two edge variables $x_{i j}$ and $x_{k l}$, the energy of a single interaction, and so on. Loop series expressions for these local observations should be very useful in improving the predictions of the mean-field cavity theory. For a graphical model with many short loops, it is desirable to represent the system as a collection of many basic clusters in the framework of Kikuchi's cluster variation method (for a review, see [17]). These basic clusters are connected to each other by joint clusters [18]. The joint clusters can be regarded as generalized edges. The present partition function loop expansion method probably is also applicable to these more complex graphical systems.

\section{Acknowledgments}

This work was finished while the authors were participating the "Interdisciplinary Applications of Statistical Physics and Complex Networks" program of KITPC (March, 2011). JQX thanks Prof. Xiang-Mao Ding for encouragements and support. This work was partially supported by the NSFC Grant 10834014, the 973-Program Grant 2007CB935903, and the PKIP Grant KJCX2.YW.W10 of CAS.

\section{Appendix A. Discrete models on a one-dimensional ring}

As a simple application of the graph expansion method discussed in the main text, we calculate the loop correction contribution for a model defined on a one-dimensional ring with $N$ vertices and $N$ edges. We assume that the edge state $x_{i, i+1}$ between two vertices $i$ and $(i+1)$ can take $Q$ different discrete values, $x_{i, i+1} \in\{1,2, \ldots, Q\}$. The energy of the ring is

$$
E=-J \delta_{x_{1,2}}^{x_{N, 1}}-\sum_{i=2}^{N-1} J \delta_{x_{i, i+1}}^{x_{i-1, i}}-J \delta_{x_{N-1, N}}^{x_{N, 1}},
$$

where $J$ is a coupling constant and $\delta_{x}^{y}$ is the Kronecker symbol. The prior distribution $\rho_{0}\left(x_{i, i+1}\right)$ is uniform over the $Q$ states.

For this simple system, the fixed point of the belief-propagation equation (16) is $q_{i \rightarrow(i+1)}(x)=1 / Q$ and $q_{(i+1) \rightarrow i}(x)=1 / Q$ for $x \in\{1,2, \ldots, Q\}$. Then we have $\Delta_{i, i+1}\left(x_{i, i+1}, x_{i+1, i}\right)=Q \delta_{x_{i, i+1}}^{x_{i+1, i}}-1$, and by a straightforward summation along the ring, the loop correction expression $(13)$ is simplified as

$$
L_{g}=(Q-1)\left[\frac{e^{\beta J}-1}{Q-1+e^{\beta J}}\right]^{N} .
$$

We notice that for $N$ being even, $L_{g} \geq 0$ and therefore the loop correction to the free energy [see $(17)$ ] is negative (the Bethe-Peierls free energy $F_{B P}$ is higher than the true 
free energy). However, if $N$ is odd and $J<0$, then $L_{g}<0$ and the loop correction to the free energy becomes positive $\left(F_{B P}\right.$ is lower than the true free energy). This different behaviour is related to the fact that, the one-dimensional ring with an odd number of interactions is frustrated when $J<0$. This simple example also shows that the loop correction $L_{g}$ decays exponentially with loop length.

\section{References}

[1] R. Brout. Statistical mechanical theory of a random ferromagnetic system. Phys. Rev., 115:824$835,1959$.

[2] G. Horwitz and H. B. Callan. Diagrammatic expansion for the ising model with arbitrary spin and range of interaction. Phys. Rev., 124:1757-1785, 1961.

[3] F. Englert. Linked cluster expansions in the statistical theory of ferromagnetism. Phys. Rev., 129:567-577, 1963.

[4] A. Georges and J. S. Yedidia. How to expand around mean-field theory using high-temperature expansions. J. Phys. A: Math. Gen., 24:2173-2192, 1991.

[5] V. Sessak and R. Monasson. Small-correlation expansions for the inverse ising problem. J. Phys. A: Math. Theor., 42:055001, 2009.

[6] M. Chertkov and V. Y. Chernyak. Loop calculus in statistical physics and information science. Phys. Rev. E, 73:065102(R), 2006.

[7] M. Chertkov and V. Y. Chernyak. Loop series for discrete statistical models on graphs. J. Stat. Mech.: Theor. Exp., page P06009, 2006.

[8] A. Montanari and T. Rizzo. How to compute loop corrections to bethe approximation. J. Stat. Mech.: Theo. Exp., page P10011, 2005.

[9] T. Rizzo, B. Wemmenhove, and H. J. Kappen. Cavity approximation for graphical models. Phys. Rev. E, 76:011102, 2007.

[10] G. Parisi and F. Slanina. Loop expansion around the bethe-peierls approximation for lattice models. J. Stat. Mech.: Theo. Exp., page L02003, 2006.

[11] V. Y. Chernyak and M. Chertkov. Loop calculus and belief propagation for $q$-ary alphabet: loop tower. In 2007 IEEE International Symposium on Information Theory, pages 316-320, 2007.

[12] M. Mézard and G. Parisi. The bethe lattice spin glass revisited. Eur. Phys. J. B, 20:217-233, 2001.

[13] S. Bounkong, J. van Mourik, and D. Saad. Coloring random graphs and maximizing local diversity. Phys. Rev. E, 74:057101, 2006.

[14] K. Y. M. Wong and D. Saad. Minimizing unsatisfaction in colourful neighbourhood. J. Phys. A: Math. Theor., 41:324023, 2008.

[15] A. Pelizzola, M. Pretti, and J. van Mourik. Palette-colouring: A belief-propagation approach. J. Stat. Mech.: Theor. Exp., page P05010, 2011.

[16] J. S. Yedidia, W. T. Freeman, and Y. Weiss. Understanding belief propagation and its generalizations. Technical report, Mitsubishi Electric Research Laboratories, 2002.

[17] A. Pelizzola. Cluster variation method in statistical physics and probabilistic graphical models. J. Phys. A: Meth. Gen., 38:R309-R339, 2005.

[18] T. Morita. Variational principle for regular and random ising models on the cactus tree or the usual lattice in the "cactus approximation". Physica A, 105:620-630, 1981. 